NOTICIAS Y COMENTARIOS 



\title{
EL COMPROMISO DE LA GEOGRAFÍA CON LA REALIDAD. XXXII CONGRESO DE LA UNIÓN GEOGRÁFICA INTERNACIONAL COLONIA, 26 A 30 DE AGOSTO DE 2012
}

\author{
Manuel Valenzuela Rubio \\ Universidad Autónoma de Madrid \\ Representante del Comité Español en la Asamblea General de la UGI, 2012
}

\section{INTRODUCCIÓN}

Bajo el título general de Down to earth (to come down to earth, bajar a la realidad) la Unión Geográfica Internacional encargó a la Asociación de Geógrafos Alemanes y a la Universidad de Colonia en la Asamblea General celebrada en Glasgow y coincidente con el XXX Congreso (2004) la organización de esta edición de sus congresos cuatrienales. Este ha sido el segundo ${ }^{1}$ congreso internacional de Geografía celebrado en territorio alemán, precisamente en una ciudad vinculada a uno de los más importantes ejes de comunicación intraeuropeos (el Rhin). Su universidad, una de las mayores y más antiguas de Alemania, fundada en 1388 como una de las cuatro universidades del Sacro Imperio Romano Germánico, contó entre sus primeros maestros con San Alberto Magno; a finales del siglo XVIII interrumpió su actividad por la invasión francesa y tras un largo período de silencio fue restablecida en 1919 gracias al empeño de uno de sus más prestigiosos alcaldes, Conrad Adenauer. Actualmente se cuenta entre las once más importantes universidades de Alemania, con seis facultades y 41.000 estudiantes. Con un millón de habitantes Colonia es la cuarta ciudad de Alemania por población y una de las más atractivas tanto para el turismo cultural, animado por la imponente catedral gótica, su colección de iglesias románicas y su variada oferta museística, musical y de ocio, como para el turismo de congresos, estimulado por una vibrante actividad nucleada en torno a su reputado complejo ferial de la margen derecha del Rhin (la Kölnmesse).

1 El anterior se remonta al año 1899, celebrado en Berlín como VII Congreso Internacional de Geografía. 


\section{LA ORGANIZACIÓN DEL CONGRESO}

La universidad de Colonia acogió en sus instalaciones todas las actividades del congreso, excluidas las reuniones específicas de las comisiones celebradas en distintas ciudades del estado federado de Renania del Norte-Wesfalia. De hecho, el Comité Organizador del Congreso fue asumido por el Instituto de Geografía de la propia universidad bajo el liderazgo de la Dra. Frauke Kraas y el profesor Dietrich Soyez. La excelente organización y el esfuerzo desplegado durante meses de preparación del congreso dieron como fruto un doble éxito de partida. En primer lugar, se superaron todas las expectativas de asistencia: más de 2.700 inscritos procedentes de 80 países, casi la mitad de ellos $(42,1 \%)$ aportados por Alemania, una cuarta parte por los restantes países de Europa $(25,4 \%$ ) y un 3,5\% por Rusia; relevante fue también la presencia de colegas procedentes del resto de Asia $(16,6 \%)$, destacando con mucho Japón y China, con más de un centenar de congresistas cada uno, seguida muy de lejos por el continente americano $(7,3 \%)$, más de un tercio de USA; peso aún más reducido tuvieron África $(2,8 \%)$ y Australia (1,9\%) (Figura 1$)$.

En segundo lugar, se consiguió una gran afluencia de jóvenes geógrafos, demostrando de ese modo que su eslogan (Down to Earth) ha tenido una importante capacidad de convocatoria sobre este segmento de colegas, tradicionalmente refractarios a participar en este tipo de eventos caros y de complicada estructura. La ubicación en recintos universitarios rompe la práctica habitual en los congresos internacionales de la UGI, en el sentido de celebrarlos en instalaciones especializadas (palacios de congresos, recintos feriales, etc.) o en hoteles, lo que tiene como efecto la profesionalización, pero también el encarecimiento de su gestión.

Es incuestionable la valentía de los colegas alemanes al optar por la autogestión del congreso, que contó con un nutrido equipo de colaboradores en las tareas de inscripción de los asistentes y de un nutrido comité científico encargado de la aún más compleja labor de

PAÍSES DE ORIGEN DE LOS PARTICIPANTES REGISTRADOS EN EL CONGRESO DE COLONIA 2012

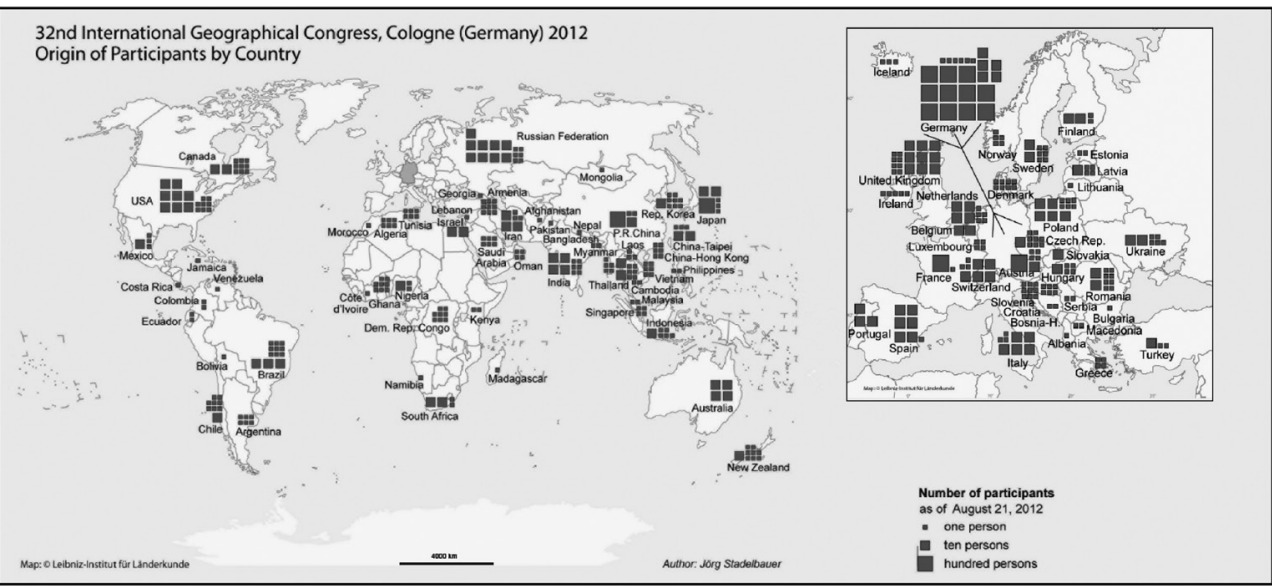

Registered participants by country of residence

Fuente: IGC Today 27/08/2012. 
evaluación y selección de la enorme masa de comunicaciones presentadas; pero, por encima de todo, es admirable el alarde de reclutamiento y de preparación del ejército de 400 voluntarios, que trabajaron duro para asegurar la recepción y atención a los congresistas y la ayuda en las cuestiones prácticas de las sesiones de trabajo. Aún con toda la buena voluntad y la disciplina germánica presentes en todas las facetas del congreso, hay que admitir ciertos desajustes e incomodidades, especialmente visibles en el uso de los espacios congresuales de una universidad con obras de reforma prácticamente omnipresentes y con una escasa oferta e idoneidad de los espacios habilitados para las exposición de editoriales y de las entidades geográficas, incluidos los comités nacionales de los distintos países presentes, entre ellos España ${ }^{2}$.

\section{LA ESTRUCTURA Y CONTENIDOS DEL CONGRESO}

Siguiendo el esquema tradicional de los congresos de la UGI, el de Colonia 2012 se componía de tres grandes grupos de actividades: las reuniones pre-congreso de las comisiones y task forces, la sesión principal y las excursiones post-congreso. Para las primeras remitimos a los colegas interesados a las respectivas páginas web de las comisiones, dada la imposibilidad de incorporar su información a esta reseña; tampoco podremos detenernos en la oferta de excursiones cortas ofertadas durante el congreso y las de mayor duración celebradas tras la conclusión de las sesión principal (26 al 30 de agosto), desarrollada en el campus de la Universidad de Colonia, literalmente ocupada por las sesiones de trabajo del congreso.

Fiel a su lema principal, Down to Erth, el Congreso de Colonia propuso a los geógrafos de todo el mundo ajustar sus contribuciones a los cuatro grandes ejes temáticos donde se ubican algunos de los mayores retos para una geografía auténticamente comprometida con los intereses y preocupaciones de los habitantes del planeta en esta etapa de globalización:

- Cambio global y globalización

- Sociedad y medio ambiente

- Riesgos y conflictos

- Urbanización y cambio demográfico

El desarrollo temático de estos ejes principales es demasiado prolijo para su reproducción aquí y, por ello, remitimos al lector interesado a la web del congreso (//igc2012.org/frontend/ index.php) o de la propia UGI (www.igu-online.org).

Todos ellos son temas sobre los que los geógrafos disponen de capacidad conceptual y metodológica con las que contribuir a dar solución científica y aplicada a tan urgentes asuntos, comprometiendo así nuestra disciplina con la realidad (bringing research down to earth). Ellos fueron los elementos estructurantes del congreso como un todo y, en particular, de la sesión principal, ya desde las propias conferencias invitadas, a cargo de ilustres miembros de nuestra comunidad científica como Dereck Gregory o ex-presidentes de la UGI (Anne Buttimer o Bruno Messerli) y de personas relevantes de otras organizaciones interesadas igualmente por alcanzar un mundo más justo y equilibrado (Club de Roma, Naciones Unidas, FAO). Creo que merece la pena enunciar los títulos precisos de las intervenciones de tan

2 De hecho, la mayoría de los 'stand' de los comités nacionales de Geografía, entre ellos el español, fueron ubicados en carpas con notables carencias ambientales y constructivas. 
notables conferenciantes, distribuidas a lo largo de todo el programa bajo la denominación de sesiones plenarias:

Klaus Töpfer (Former Federal Minister of Environmental Affairs and Former Director of UNEP),On the way to the Anthropocene. Consequences for scientific research, societal understanding and political responsibility.

Anne Buttimer (Geographer, IGU Past President, University College Dublin, Dublin, Ireland), Diverse perspectives on society and environment: retrospect and prospect.

Martin Lees (Former Secretary General, Club of Rome), Demographic change and urbanisation within the boundaries of a fragile planet.

Surinder Aggarwal (Geographer, University of Delhi, New Delhi, India), Emerging global urban order and challenges for harmonious urban development.

Eduardo de Mulder (Initiator and Executive Director of the UN International Year of Planet Earth), Global planetary change and human globalization.

Bruno Messerli (Geographer, University of Bern, Bern, Switzerland), Global change and globalisation - challenges for Geography.

Stephan Bass (Natural Resources Officer in the Climate, Energy and Tenure Division, FAO Rome), Risk and conflicts.

Derek Gregory (Geographer, University of British Columbia, Vancouver, Canada), Deadly embrace: war, distance and intimacy. ${ }^{3}$

El desarrollo del Congreso tuvo como armazón fundamental las 144 sesiones dedicadas a la presentación de comunicaciones referidas a los cuatro grandes temas arriba enunciados, seleccionadas por el Comité Científico mediante un proceso muy competitivo. En dos sesiones monográficas muy concurridas se concentraron la presentación y discusión de pósters, objeto de una convocatoria diferenciada que mereció una entusiasta respuesta por parte de jóvenes geógrafos; el resultado fue una colección de 180 posters distribuidos entre los cuatro grandes temas del congreso, de la que salieron los cuatro premiados, uno por cada tema. También los estudiantes tuvieron asignadas sesiones especiales para dar a conocer sus proyectos científicos en dos formatos: presentaciones rápidas o pósters. Incluso los estudiantes de Geografía procedentes de 40 países tuvieron la oportunidad de participar en el congreso por mediación de la Olimpiada de la UGI (iGeo). Su presencia en diversos puntos de la ciudad realizando trabajos para la competición aportó al congreso un sesgo juvenil muy estimulante.

Junto a las anteriores modalidades de participación, el Congreso de Colonia también dio entrada a un Foro de Jóvenes Investigadores dirigido a jóvenes docentes universitarios, postgraduados, doctorandos y similares. Este foro tuvo una estructura muy particular pues se componía de una cuota reservada a este colectivo en las sesiones ordinarias, además de un taller de carácter formativo con duración de un día, celebrado antes del congreso. Por último, el programa del congreso incluía dos simposios en alemán sobre Didáctica de la Geografía y Geografía Aplicada, organizados por las asociaciones especializadas en dichas temáticas.

En una sesión especial se realizó una presentación monográfica sobre el estado en que se encuentra la iniciativa de la UGI para conseguir la proclamación por parte de la ONU de

3 Todas las intervenciones están disponibles en www.igu-online.org 
un Año Internacional de la Conciencia Global (Global Understanding), mediante el cual se pretende dar continuidad a iniciativas como el Año Internacional de la Montaña, el de la Biodiversidad o el del Planeta Tierra. En este caso la base justificativa la aporta la necesidad de reconciliar las escalas local y global en todos los aspectos de la vida sobre el planeta, lo que requiere una simbiosis entre ciencia y vida cotidiana. Para conseguirlo se aspira mediante esta iniciativa a incrementar la conciencia social utilizando para ello toda una batería de medios escritos, informáticos, multimedia, etcétera. De momento, se ha montado una organización de apoyo a esta iniciativa, coordinada desde el Departamento de Geografía de la Universidad de Jena, en Alemania.

Formando parte del programa oficial del Congreso de Colonia, pero en una sesión especial celebrada el domingo 26 de agosto, tuvieron lugar dos reuniones organizadas por la Sociedad Europea para la Geografía (EUGEO) y decididas por los nuevos presidente y secretario de la Sociedad, Henk Ottens (Real Sociedad Geográfica de los Paises Bajos) y Maximiliano Tabusi (Sociedad Geografica Italiana), respectivamente, dentro del programa aprobado en el III Congreso de Geografía de Europa celebrado en Londres el año 2011 y consistente en organizar cada año un evento en forma de congreso, conferencia o seminario. En 2012 el evento ha consistido en un seminario sobre El Estado de la Geografía en los Países Europeos, con la vista puesta en el fortalecimiento de la presencia de nuestra disciplina en cuantos ámbitos ya se encuentra reconocida (enseñanza en todos los niveles, en la investigación, en el ejercicio profesional o en los medios); para conseguirlo EUGEO propone involucrar de forma individual o colectiva a las sociedades geográficas de Europa. Entre los doce miembros de EUGEO que realizaron presentaciones sobre sus respectivos países, el Estado de la Geografía en España fue asumido por el autor de esta reseña, representante de la Real Sociedad Geográfica, miembro fundador de EUGEO. El segundo evento de la jornada fue la Asamblea General de EUGEO, en cuya agenda, entre otros asuntos ordinarios destacó la presentación del programa provisional del IV Congreso de Geografía de Europa, a celebrar en Roma en septiembre de 2013.

\section{LA ASAMBLEA GENERAL DE LA UNIÓN GEOGRÁFICA INTERNACIONAL}

Simultáneamente a la celebración de las sesiones del Congreso tuvo lugar, como es habitual, la Asamblea General de la UGI, máxima fuente de legitimidad de la organización y foro en que se toman las grandes decisiones, básicamente corporativas, lo que para muchos explica la relativamente poca incidencia que sobre el devenir mundial de nuestra disciplina llegan a tener. Para comenzar, la presencia de las delegaciones está fuertemente condicionada por la situación económica de los países miembros y por su lejanía a la sede del congreso. Así, de los 48 comités presentes en la Asamblea, sólo dos representaban a países africanos (Nigeria y Zimbabwe) y otros tantos a hispanoamericanos (Chile y México).

En el capítulo de decisiones más relevantes de la Asamblea se aprobaron, en primer lugar, para el próximo período entre congresos las comisiones y task forces que se mantienen del período anterior, las que decaen y las de nueva creación; el resultado se recoge en el cuadro 1. No debe olvidarse que las comisiones en la UGI son las encargadas de mantener entre dos congresos el impulso investigador mediante sus reuniones periódicas, muchas veces anuales. 
Cuadro 1

COMISIONES Y TASK FORCES APROBADAS PARA EL PERIODO 2012-2016, PRESIDENTES Y PAÍS DE ORIGEN

\begin{tabular}{|c|c|c|c|}
\hline Code & Commission & Chair & Country \\
\hline C12-01 & Applied Geography & Graham Clarke & United Kingdom \\
\hline $\mathrm{C} 12-02$ & $\begin{array}{l}\text { Arid Lands, Humankind, } \\
\text { Environment }\end{array}$ & Mahmoud Ashour & Egypt \\
\hline C12-03 & Biogeography and Biodiversity & Udo Schickhoff & Germany \\
\hline C12-04 & Climatology & Zbigniew Ustrnul & Poland \\
\hline $\mathrm{C} 12-05$ & Coastal Systems & Edward Anthony & France \\
\hline C12-06 & Cold Regions Environments & $\begin{array}{l}\text { Nancy Doubleday } \\
\text { Dario Trombotto }\end{array}$ & \begin{tabular}{|l|} 
Canada \\
Argentina \\
\end{tabular} \\
\hline C12-07 & Cultural Approach in Geography & Benno Werlen & Germany \\
\hline C12-08 & Dynamics of Economic Spaces & Neil Reid & USA \\
\hline C12-09 & Environmental Evolution & Tatjana Boettger & Germany \\
\hline $\mathrm{C} 12-10$ & Gender and Geography & Shirlena Huang & Singapore \\
\hline C12-11 & Geographical Education & $\begin{array}{l}\text { Joop van der Schee } \\
\text { John Lidstone }\end{array}$ & $\begin{array}{l}\text { Netherlands } \\
\text { Australia } \\
\end{array}$ \\
\hline $\mathrm{C} 12-12$ & Geographical Information Science & Francis Harvey & United States \\
\hline C12-13 & Geography of Governance & Jan Bucek & Slovak Republic \\
\hline C12-14 & $\begin{array}{l}\text { Geography of the Global } \\
\text { Information Society }\end{array}$ & Mark Wilson & USA \\
\hline C12-15 & $\begin{array}{l}\text { Geography of Tourism, Leisure, } \\
\text { and Global Change }\end{array}$ & Dieter Muller & Sweden \\
\hline C12-16 & GeoParks & Dongying Wei & China-Beijing \\
\hline C12-17 & $\begin{array}{l}\text { Global Change and Human } \\
\text { Mobility }\end{array}$ & $\begin{array}{l}\text { Josefina Domínguez } \\
\text { Mujica }\end{array}$ & Spain \\
\hline $\mathrm{C} 12-18$ & Hazard and Risks & Shigeko Haruyama & Japan \\
\hline C12-19 & Health and Environment & $\begin{array}{l}\text { Wuyi Wang } \\
\text { Thomas Krafft } \\
\end{array}$ & $\begin{array}{l}\text { China-Beijing } \\
\text { The Netherlands }\end{array}$ \\
\hline $\mathrm{C} 12-20$ & History of Geography & Jacobo Garcia Alvarez & Spain \\
\hline C12-21 & $\begin{array}{l}\text { Indigenous Peoples, Knowledges } \\
\text { and Rights }\end{array}$ & Brad Coombes & New Zealand \\
\hline $\mathrm{C} 12-22$ & Islands & Chang-yi David Chang & China-Taipei \\
\hline $\mathrm{C} 12-23$ & Karst & Elena Trofimova & Russia \\
\hline C12-24 & $\begin{array}{l}\text { Land Degradation and } \\
\text { Desertification }\end{array}$ & Paul Hudson & USA \\
\hline C12-25 & $\begin{array}{l}\text { Landscape Analysis and } \\
\text { Landscape Planning }\end{array}$ & Nodar Elizbarashvili & Georgia \\
\hline C12-26 & Land Use and Land Cover Change & Ivan Bicík & Czech Republic \\
\hline $\mathrm{C} 12-27$ & Latin American Studies & Juan Manuel Delgado & Peru \\
\hline C12-28 & Local Development & Michael Sofer & Israel \\
\hline
\end{tabular}




\begin{tabular}{|c|c|c|c|}
\hline Code & Commission & Chair & Country \\
\hline C12-29 & $\begin{array}{l}\text { Marginalization, Globalization, } \\
\text { and Regional and Local Responses }\end{array}$ & Stanko Pelc & Slovenia \\
\hline $\mathrm{C} 12-30$ & Mediterranean Basin & Maria Paradiso & Italy \\
\hline C12-31 & Modelling Geographical Systems & Yee Leung & $\begin{array}{l}\text { China-Hong } \\
\text { Kong }\end{array}$ \\
\hline C12-32 & $\begin{array}{l}\text { Mountain Response to Global } \\
\text { Change }\end{array}$ & Joerg Loeffler & Germany \\
\hline C12-33 & Political Geography & Elena Dell'Agnese & Italy \\
\hline $\mathrm{C} 12-34$ & Population and Vulnerability & Etienne Piguet & Switzerland \\
\hline C12-35 & Sustainability of Rural Systems & $\begin{array}{l}\text { Ana Firmino } \\
\text { Kim Doo-Chul }\end{array}$ & $\begin{array}{l}\text { Portugal } \\
\text { Japan }\end{array}$ \\
\hline C12-36 & Toponymy & Cosimo Palagiano & Italy \\
\hline C12-36 & $\begin{array}{l}\text { Transformation Processes in } \\
\text { Megacities }\end{array}$ & Frauke Kraas & Germany \\
\hline C12-38 & Transport and Geography & Richard Knowles & United Kingdom \\
\hline C12-39 & $\begin{array}{l}\text { Urban Geography:Urban } \\
\text { Challenges in a Complex World }\end{array}$ & Celine Rozenblat & Switzerland \\
\hline C12-40 & Water Sustainability & Claudio Cassardo & Italy \\
\hline Code & Task Force & Chairperson & Country \\
\hline $\begin{array}{l}\text { TF12- } \\
01\end{array}$ & Olympiad & $\begin{array}{l}\text { Henk Ankone } \\
\text { Joop van der Schee }\end{array}$ & $\begin{array}{l}\text { The Netherlands } \\
\text { The Netherlands }\end{array}$ \\
\hline
\end{tabular}

Fuente: www.ugi-online.org

Nota: El nombre de las comisiones se mantiene en inglés para evitar cambiar su numeración oficial.

Los cambios introducidos respecto a las aprobadas en el Congreso de Túnez de 2008 no son significativos ya que tan solo una comisión ha sido cancelada (Retos Geomorfológicos para el siglo XXI) frente a cuatro de nueva creación: Toponimia, Cuenca Mediterránea, Estudios Latinoamericanos y Transformación de las Megaciudades. Entre los que se mantienen, pero cambian de presidente, citaremos la comisión de Geografía del Turismo, Ocio y Cambio Global, en la que Sakko Saarinen, promovido a una vicepresidencia de la UGI, es sustituido por el sueco Dieter Muller; la de Geografia Urbana, presidida hasta ahora por el danés Christian Mattiessen, da paso a la profesora de la Universidad de Lausanne Celine Rozenblat, aunque la que más interés nos despierta es la comisión de Cambio Global y Movilidad Humana, cuya nueva presidenta es nuestra compatriota de la universidad de Las Palmas Josefina Domínguez Mújica, en sustitución del italiano Armando Montanari. También es digna de cita la continuidad de Jacobo García Álvarez, profesor de la universidad Carlos III de Madrid, al frente de la comisión de Historia de la Geografía, que afronta su segundo mandato cuatrienal. También ha decaído la task force sobre Megaciudades.

En el capítulo de nuevos países miembros de la UGI se aprobaron la candidatura de Kuwait y de la Autoridad Nacional Palestina, en este caso con algunas reservas por tratarse de un territorio que para muchos delegados no cumple el requisito de tener estatus de Estado. 
En cuanto a su situación presupuestaria, el balance de las cuentas de la organización a fecha de diciembre de 2011 podía calificarse como muy favorable, dado que arrojaba un superavit de 23.737 dólares USA.

En el ámbito organizativo, mediante votación de los delegados de los países presentes en la Asamblea, se produjo la elección del nuevo presidente y de cuatro vicepresidentes para el período 2012-2016 entre los diez candidatos aspirantes, con el siguiente resultado:

Presidente: Vladimir Kolosov, Academia de Ciencias de Rusia (Moscú), que sustituye al norteamericano Ron Adler.

Vicepresidentes: R.S. Singh, Universidad de Nueva Delhi (India)

Dietrich Soyez, Universidad de Colonia (Alemania)

Joos Droogleever-Fortuijn, Universidad de Amsterdam (Paises Bajos)

Jarkko Saarinen, Universidad de Oulu (Finlandia)

Con la entrada de los nuevos vicepresidentes y la no presentación a la reelección de la vicepresidenta mexicana Irasema Alcántara (UNAM), además de la salida del italiano Giuliano Bellezza, se da una situación inédita en el nuevo comité, a saber, la falta de representación en el Comité Ejecutivo de la UGI no sólo de la geografía hispanohablante, sino de toda la comunidad de geógrafos que se expresan en alguna de la lenguas de ascendencia latina. Este hecho deberá ser muy tenido en cuenta para futuras renovaciones del Comité.

En el capítulo de toma en consideración por la Asamblea de los países organizadores de los futuros eventos internacionales de la UGI, el proceso de elección del país organizador de la Conferencia Regional de 2018 entre los dos candidatos presentados (Taiwan y Perú) no pudo ser culminado por decisión del Comité Ejecutivo, que acordó posponer la decisión hasta tanto no tuviera información adicional sobre ambas candidaturas; por tanto, la elección quedo en suspenso. Por el contrario, fue aceptada por unanimidad la candidatura del Comité Nacional de Turquía como país organizador del Congreso Internacional del año 2020. Así mismo, se tomó en consideración por la Asamblea la celebración de un Congreso Internacional extraordinario en 2022 con ocasión del 150 aniversario de la fundación en 1922 de la Unión Geográfica Internacional y del primer congreso internacional celebrado en Amberes en 1921. La propuesta fue aprobada sin lugar para su celebración.

Por tanto, el calendario y lugar de celebración de las futuras reuniones convocadas por la UGI quedó como sigue:

- 2013 Conferencia Regional de Japón en Kyoto

- 2014 Conferencia Regional de Polonia en Cracovia

- 2015 Conferencia Regional de Rusia en Moscú

- 2016 Congreso Internacional de China en Beijing

- 2018 Conferencia Regional (elección del organizador pospuesta)

- 2020 Congreso Internacional de Turquía en Estambul

- 2022 Congreso Internacional del 150 Aniversario (Pendiente el organizador)

Estos fueron los acuerdos más relevantes de la Asamblea, pero no podemos dejar de citar otros dos asuntos de contenido muy desigual. El uno despierta un sentimiento de admiración por tratarse de premios concedidos por la UGI a varias personalidades beneméritas por su aportación relevante al progreso de la Geografía mundial; en tal sentido fue galardonado con 
la IGU Planet and Humanity Medal Mr. Lester Brown, presidente e investigador principal del Earth Policy Institute de Washington; paralelamente, se premió como Laureados de Honor a los siguientes geógrafos: Larry S. Bourne (Canadá), Mohammed S.I. Makki (Arabia Saudî), Janice J. Monk (USA) y a Hiroshi Tanabe (Japón). El reverso triste de la moneda lo aportó la definitiva cancelación de la Home of Geography, ubicada en la Villa Celimontana, Roma, tradicional sede de la Societá Geográfica Italina, por el efecto combinado del cese de la financiación aportada por la UGI y por el Estado italiano; al mismo tiempo ha cesado como vicepresidente de la UGI el creador y alma de la ejemplar institución geográfica, el profesor Giulliano Bellezza.

Es de destacar entre las cuestiones suscitadas a lo largo de la Asamblea la presentación de los resultados del grupo de trabajo creado en 2010 e integrado por diversos geógrafos de la Universidad de Hunan (Changsha, China) y de la Universidad de Ámsterdam (profesor Ton Dietz), cuya finalidad era desarrollar un proyecto piloto centrado en la creación de un sitio web en torno a la cuestión de las ciudades sostenibles. Concebido como el buque insignia de la UGI para marcar el rumbo sobre la manera de crear redes de información en torno a determinados temas relevantes de la actualidad mundial impulsadas por geógrafos, la Global Sustainable Cities Information Network (GSCIN) ha tomado cuerpo ya en dos páginas web, la una sobre el caso de la ciudad china de Changsha (http://zh.oursus.org) y la otra con una síntesis del caso chino pero también con ejemplos de otras ciudades el mundo (www.oursus.org).

Recogemos también en esta nota otra información de gran utilidad práctica para los geógrafos españoles: la presentación de la recién creada base de datos de publicaciones periódicas, actualmente ya accesible desde la web de la UGI (www.igu-online.org); impulsada igualmente por el profesor Dietz y otros colegas de la Universidad de Ámsterdam; a través de ella están accesibles más de 1.000 revistas geográficas de todo el mundo.

\section{LA PRESENCIA EN COLONIA DEL COMITÉ ESPAÑOL DE LA UGI}

En primer lugar, hay que admitir que la presencia física de la Geografía española visualizada a través de un stand se ha visto seriamente devaluada por el efecto combinado de la merma presupuestaria y de la paralela reducción de la oferta de espacios por parte de los organizadores. Lo cual ha determinado que ni de lejos se haya repetido, ni por cantidad de espacio ni por los medios materiales y humanos disponibles, el precedente de las tres ediciones del Congreso Internacional de la UGI celebradas desde el año 2000 (Seúl, Glasgow y Túnez). Aún así, el modesto stand de cinco metros cuadrados, sin paredes ni estructura fija y con tan sólo una mesa y dos sillas como único mobiliario, ha sido el punto de encuentro de los geógrafos españoles presentes en Colonia y ha dado cara y voz a la Geografía española ante la comunidad geográfica internacional. La rentabilidad de la reducida inversión realizada en el alquiler de este espacio ha sido sin duda elevada, pues ha permitido al Comité Español de la UGI y a las entidades en él representadas (básicamente la Real Sociedad Geográfica, la Asociación de Geógrafos Españoles, la Societat Catalana de Geografía y el Instituto Geográfico Nacional) proyectar la imagen de unidad y diversidad que nos caracteriza. A ello colaboraron los miembros del comité presentes en Colonia, que aseguraron, con la ayuda ocasional de algunos colegas asistentes al congreso, la apertura permanente del stand 
Figura 2

INSTALACIONES DE LA UNIVERSIDAD DE COLONIA OCUPADAS POR LOS GEÓGRAFOS DEL CONGRESO INTERNACIONAL DE LA UGI 2012

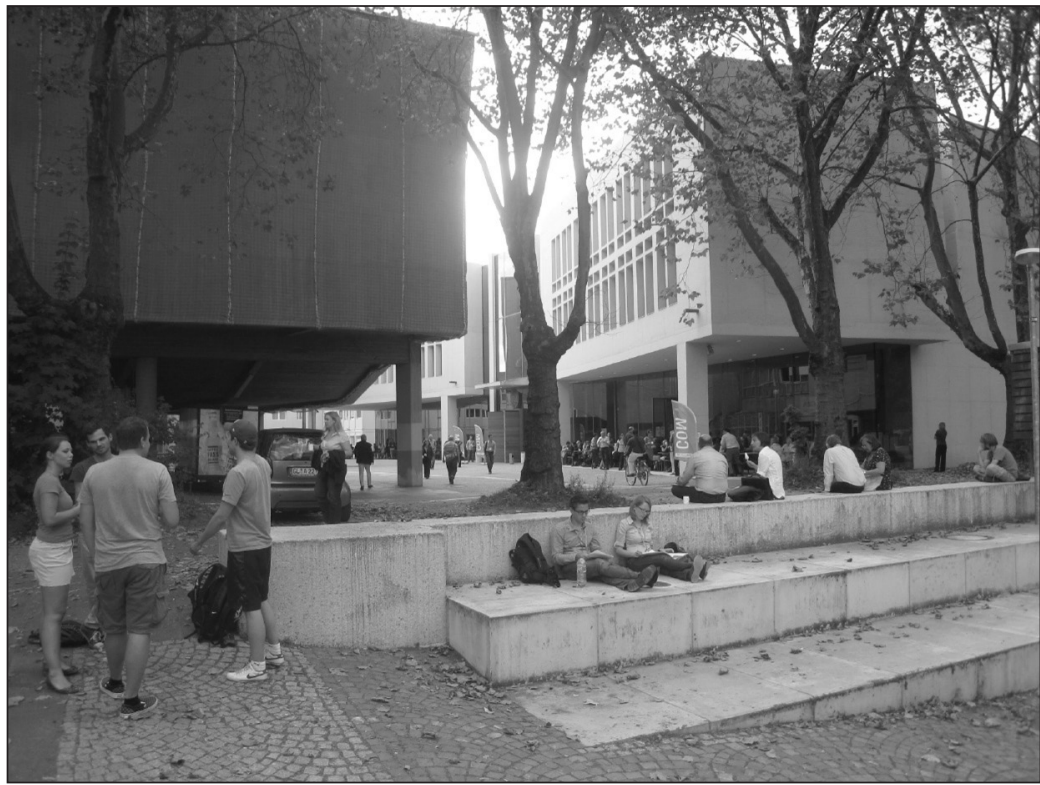

Figura 3

EL STAND DEL COMITÉ ESPAÑOL DE LA UGI, PUNTO DE ENCUENTRO DE LOS GEÓGRAFOS ESPAÑOLES ASISTENTES AL CONGRESO DE COLONIA

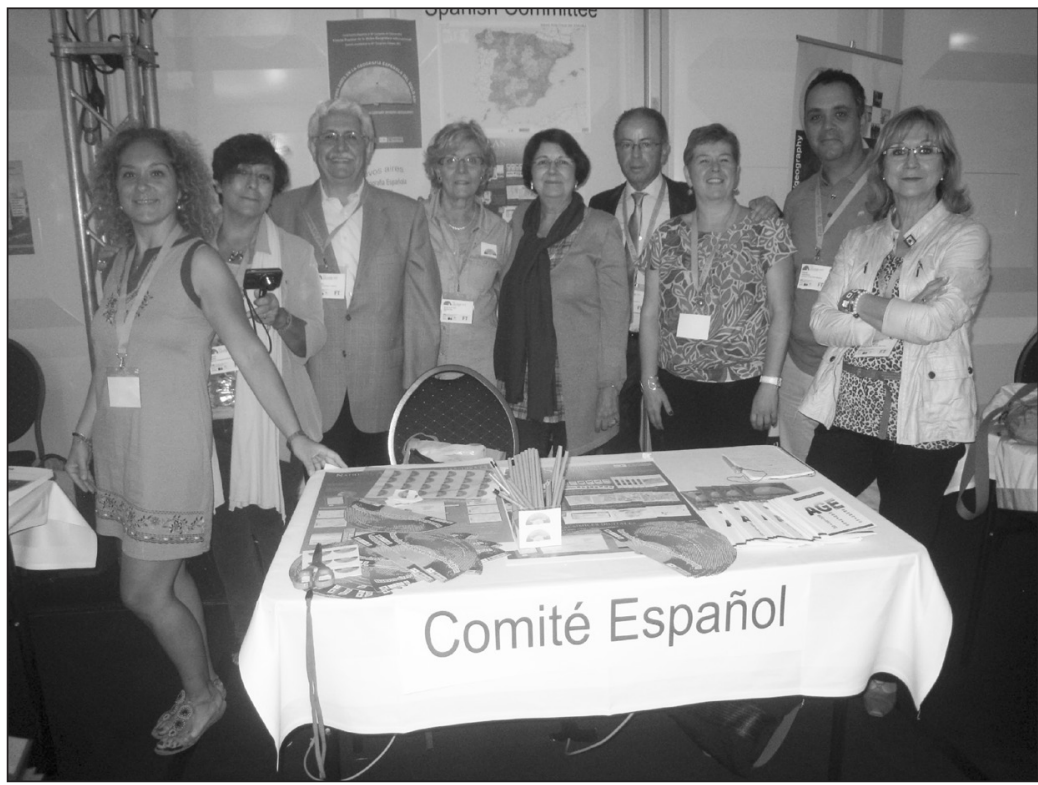


y la fluidez de la información a los visitantes interesados por España, la geografía española o por las entidades integradas en el comité español de la UGI.

Merece la pena resaltar en esta nota dos de las tareas desarrolladas en el stand español en Colonia: la información a los visitantes sobre España y la geografía española y la distribución de la Aportación Española al XXXII Congreso Internacional de la UGI, ambas íntimamente ligadas, ya que sin duda el atractivo mayor y casi único de nuestro stand en esta ocasión consistió justamente en la distribución del CD elaborado durante los dos últimos años como forma de proyección de la geografía española en este escenario internacional.

Como ya conocen sin duda todos los miembros de las organizaciones integrantes del comité a través de las comunicaciones distribuidas en su momento, se ha continuado también en esta ocasión una tradición de décadas a la hora de elaborar la aportación de la geografía española al congreso de Colonia, consistente en la creación dentro del comité de una comisión editorial ad hoc, encargada de impulsar y dirigir la elaboración de un volumen con diversas aportaciones científicas de los geógrafos españoles en torno a los ejes temáticos propuestos por los organizadores del congreso, ya reseñados más arriba. A tal fin y como primera providencia se difundió una convocatoria abierta entre los geógrafos españoles solicitando sus propuestas, al mismo tiempo que se comprometía a un grupo de autores invitados con asignación de temas previamente seleccionados por el comité científico y editorial. Como complemento del volumen de textos se realizó, con apoyo del CSIC y de una empresa privada, una Bibliografía Geográfica Española (2000-2012), concebida como el primer paso para la creación de una base de datos con la producción científica de los geógrafos españoles en la primera década del siglo XXI.

La publicación ${ }^{4}$ con las contribuciones científicas de los geógrafos españoles al Congreso de Colonia ha sido editada en esta ocasión y por primera vez sólo en soporte digital ${ }^{5}$ con sendas versiones en español e inglés constando, como ya se ha señalado, de dos grandes bloques de textos: el primero recoge las aportaciones de los autores invitados y el segundo las aportaciones a la convocatoria libre entre los geógrafos españoles tras superar la oportuna evaluación. El índice esquemático de la obra es el que sigue:

Presentación: Juan Velarde Fuertes

Autores Invitados:

BOSQUE SENDRA, J. (Universidad de Alcalá de Henares): Las TIG y los modelos de simulación para el estudio del cambio global.

DOMÍNGUEZ MÚJICA, J. (Universidad de Las Palmas de Gran Canaria): El desafío científico y social de la inmigración extranjera en España.

MARTÍN VIDE, J. (Universidad de Barcelona): Los límites del planeta, el cambio climático y la gobernanza.

4 COMITÉ ESPAÑOL DE LA UNIÓN GEOGRÁFICA INTERNACIONAL (2012) Nuevos aires en la Geografía Española (New trends in the XXI Century Spanish Geography). Contribución Española al XXXII Congreso de Unión Geográfica Internacional. Madrid, Comité Español de la UGI, 612 págs. El Consejo Editorial de la obra ha estado formado por Teresa Albert, $\mathbf{M}^{\mathrm{a}}$ Carmen Cañizares, Juan Antonio Cebrián, $\mathrm{M}^{\mathrm{a}}$ José Lozano, Mª Asunción Martín-Lou, Carlos Manuel Valdés y Manuel Valenzuela. La producción general y la edición del libro corrió a cargo de Teresa Albert y Laura Carrasco con la colaboración de su equipo del Instituto Geográfico Nacional (Diego Gómez, David Tapiador, Manuel Avendaño y Fernando González)

5 Las razones son tanto de tipo económico y presupuestario como de carácter práctico, dada la dificultad de distribución que un libro en papel tiene, incluso siendo gratuito como sería el caso. 
MORAL ITUARTE, L. del (Universidad de Sevilla); Crisis del capitalismo global y medio ambiente.

OLCINA CANTOS, J. (Universidad de Alicante): Globalización y sostenibilidad: retos ambientales de un mundo globalizado.Una visión desde la geografía española..

SAURI PUJOL, D. (Universidad Autónoma de Barcelona): Riesgos y conflictos socioambientales.

VALENZUELA RUBIO, M. (Universidad Autónoma de Madrid): Progresos hacia un modelo urbano español más sostenible en el siglo XXI.

\section{Otras contribuciones:}

ALMOGUERA, P. (Universidad de Sevilla): Transformaciones recientes en el tejido urbano de las capitales andaluzas: génesis y desarrollo de barrios de inmigrantes.

BAYONA, J.; GIL, F. y PUJADAS, I. (Universidad de Barcelona): La evolución demográfica de las áreas metropolitanas españolas: entre el boom migratorio y la crisis económica e inmobiliaria.

BENITO DEL POZO, P. (Universidades de León) y PASCUAL, H. (Universidad de Valladolid): Innovación y territorio: los parques tecnológicos españoles a la luz de la experiencia en EE.UU.

DELGADO VIÑAS, C. (Universidad de Cantabria): Impacto de la urbanización extensiva sobre los espacios protegidos españoles.

DÍEZ, R. (Universidad de Granada) y SANCHEZ ESCOLANO, L.M. (Universidad Complutense de Madrid): Cuando la política ignora la gobernanza. Riesgos, conflictos e intereses en el fracaso del aeropuerto de Ciudad Real.

FERNÁNDEZ GARCIA, F. (Universidad Autónoma de Madrid) y RASILLA, D. (Universidad de Cantabria): Estrés térmico e influencia urbana en el área metropolitana de Madrid.

LÁZARO, M.L. (Universidad Complutense) y GONZÁLEZ, M.J. (Universidad de León): El incierto futuro del paisaje de dehesa y su gestión integral sostenible.

LOIS, R. y PIÑEIRA, M.J. (Universidad de Santiago de Compostela): Población, crecimiento urbano y mercado de la vivienda.

MÉNDEZ, R. (CSIC); SANCHEZ, S. y PRADA, J.(Universidad Complutense): El fenómeno de las 'shrinking cities'en España : una aproximación a las causas, efectos y estrategias de revitalización a través del caso de Avilés.

MORILLAS, M.; FRECH, X.; MARTÍ-HENNENBERG, J. (Universidad de Lleida) y GARCÍA, A. (Fundación de Ferrocarriles): Transformación urbana y desarrollo del ferrocarril en España 1850-2000.

PRECEDEO, A. (Universidad de Santiago); MÍGUEZ, A. (Escuela de Magisterio ESCUNI) y OROSA, J. (Universidad de A Coruña): La gestión y planificación de los espacios supramunicipales: de las áreas metropolitanas a las regiones urbanas.

RODRÍGUEZ, R.; MIRANDA, M.; ÁLVAREZ, M.; DÍEZ, A. (Universidad Politécnica de Madrid) y PAPI, F. (Instituto Geográfico Nacional): Modelización urbana $3 D$ a partir de datos obtenidos con LIDAR e imágenes de alta resolución. 
VIDAL, M.J. (Universidad Autónoma de Madrid) y FERNÁNDEZ PORTELA, J. (Universidad de Valladolid): Crecimiento metropolitano y nuevas tipologías residenciales en la ciudad de Madrid (España).

Cuando se redacta esta reseña, el CD con la aportación española a Colonia ha sido ya distribuido a las entidades integrantes del Comité Español de la UGI y a los miembros de la Asociación de Geógrafos Españoles y de la Real Sociedad Geográfica. También está disponible en las respectivas páginas webs y en la del Instituto Geográfico Nacional. Por otra parte, la distribución del CD entre los visitantes del stand fue una de las tareas prioritarias asumidas por los miembros del comité y otros geógrafos colaboradores a lo largo del congreso, a donde se enviaron 400 ejemplares; la mitad de ellos fueron entregados en mano a los visitantes del stand, un tercio a los miembros de las comisiones y sesiones de trabajo, con preferencia a los miembros permanentes de las comisiones y presidentes de las sesiones, y el cuarto restante a los delegados de los comités nacionales presentes en la Asamblea General, incluidos los miembros salientes y entrantes del Consejo Ejecutivo de la UGI, tarea que realizó el propio representante del comité español en la Asamblea y autor de esta reseña.

Insistiremos algo más sobre el papel catalizador que ejerció el stand del comité español en el congreso de Colonia, en gran medida debido en esta ocasión a la entrega gratuita del CD con los textos de la aportación española. De ello da fe el número visitantes y su dispersión territorial y académica. Gracias a un registro individualizado que de todos los receptores del CD se llevó a cabo a lo largo del congreso, tenemos un cálculo bastante exacto de los congresistas más interesados por la geografía española: 186, lo que viene a representar alrededor de $7 \%$ del total de los inscritos en el congreso. De la explotación realizada para esta reseña del Censo de Visitantes del stand hemos obtenido algunos resultados elocuentes. Comenzaremos por su distribución por continentes, de la que se desprende que, como era de suponer, más de la mitad de nuestros visitantes procedían de países europeos $(53,3 \%)$, más del doble que los asiáticos $(25,5 \%)$, seguidos a muy larga distancia por los suramericanos $(7,5 \%)$ y los rusos $(5,3 \%)$; pero, aunque pocos, también se interesaron por nuestro stand siete norteamericanos, cinco africanos e incluso dos australianos. Ahora bien, la sorpresa salta cuando analizamos los países de procedencia y vemos cómo, entre los que aportan más de cinco visitantes, destacan con mucho, como era lógico, los alemanes, que totalizan ellos solos el 17,2\% de las visitas, pero a continuación no aparece otro país europeo, sino India, que con 13 visitantes representa el 7\% del censo; a continuación vienen Portugal y Rusia, empatados a 10 visitantes; hay que esperar a la cuarta posición para encontrar a los visitantes españoles (excluidos, claro está, los miembros del comité), igualados con los chinos en ocho visitantes; muy de cerca le siguen Chile, Francia e Irán con seis e Italia, Rumanía y Japón con cinco (cuadro 2 y figura 3 )

También nos interesamos por las universidades a las que pertenecían nuestros visitantes y el resultado a nivel de grande números ha sido de 134; el desglose recogido en el cuadro 3 y la figura 2 no altera sustancialmente en cuanto a porcentajes la distribución por países, siendo de destacar el caso de Alemania con un 17,2\% de las universidades de procedencia de los visitantes, destacando aún más sobre los restantes países. Siendo la dimensión cuantitativa en términos numéricos un indicador importante de la proyección de la geografía española en Colonia, no es menos digno de ser tenido en cuenta la categoría de un nutrido 
Cuadro 2

PRINCIPALES PAISSES DE PROCEDENCIA DE LOS VISITANTES Y DE SUS UNIVERSIDADES AL STAND DEL COMITÉ ESPAÑOL DE LA UGI

\begin{tabular}{|l|c|c|c|c|}
\hline \multicolumn{1}{|c|}{ Países } & Visitantes & Universidades & \% [2] & \% [3] \\
\hline Alemania & 32 & 20 & 17,2 & 14,9 \\
\hline India & 13 & 10 & 7,0 & 7,5 \\
\hline Portugal & 10 & 7 & 5,4 & 5,2 \\
\hline Rusia & 10 & 6 & 5,4 & 4,5 \\
\hline China & 8 & 6 & 4,3 & 4,5 \\
\hline España & 8 & 7 & 4,3 & 5,2 \\
\hline Chile & 6 & 4 & 3,2 & 3,0 \\
\hline Irán & 6 & 5 & 3,2 & 3,7 \\
\hline Francia & 6 & 4 & 3,2 & 3,0 \\
\hline Italia & 5 & 5 & 2,7 & 3,7 \\
\hline Rumania & 5 & 3 & 2,7 & 2,2 \\
\hline Japón & 5 & 3 & 2,7 & 2,2 \\
\hline Total & $\mathbf{1 8 6}$ & $\mathbf{1 3 4}$ & $\mathbf{1 0 0 , 0}$ & $\mathbf{1 0 0 , 0}$ \\
\hline
\end{tabular}

Fuente: Censo de visitantes al stand del Comité Español de la UGI (Elaboración propia).

Nota: Los porcentajes están calculados sobre el total del Censo de 186 visitantes.

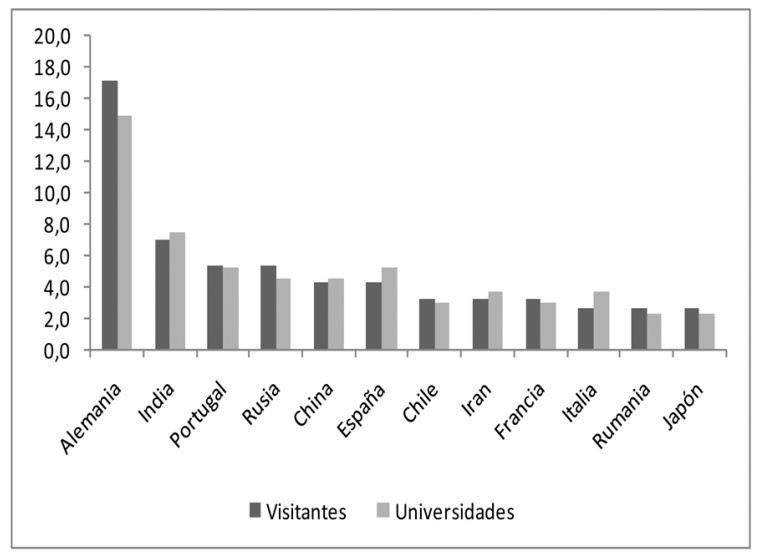

grupo de nuestros visitantes, entre los que, sin ánimo de exhaustividad, citaremos aquí algunos de los más significados como el expresidente de la UGI Bruno Messerli, Jean Robert Pitte, exrector de La Sorbona y presidente de la Sociedad de Geografía de Paris, Christian Pierret, presidente-fundador del Festival Internacional de Geografía, Armando Montanari, catedrático de La Sapienza y vicepresidente de EUGEO o el recién elegido vicepresidente de la UGI, el profesor R.S. Singh de la Universidad de Nueva Delhi. 


\section{LA PRESENCIA Y PARTICIPACIÓN DE LOS GEÓGRAFOS ESPAÑOLES EN LAS SESIONES DEL CONGRESO DE COLONIA}

No podemos dar comienzo a este epígrafe sin felicitarnos del incremento que ha experimentado en este congreso la presencia de geógrafos españoles, tanto por el número de los inscritos como por la cantidad y calidad de las aportaciones a las sesiones de trabajo ${ }^{6}$. Sobre los inscritos en el Congreso de Colonia por el país de residencia la única fuente disponible actualizada es el mapa reproducido en el apartado 2 de esta nota en el que aparece España con 62 inscritos, un record respecto a congresos anteriores reseñados por el autor de este texto. Más elocuencia que el número de inscritos tiene, a los efectos de nuestro análisis, la presentación de comunicaciones y aquí el esfuerzo realizado descrito en la nota 5 adquiera todo su significado pues para conseguirlo ha habido que hacer una tediosa búsqueda a través

Cuadro 3

COMUNICACIONES PRESENTADAS AL CONGRESO DE COLONIA POR UNIVERSIDADES

\begin{tabular}{|l|c|c|c|}
\hline Universidades [1] & $\begin{array}{c}\mathbf{N}^{\circ} \text { de autores } \\
{[\mathbf{2}]}\end{array}$ & $\begin{array}{c}\mathbf{N}^{\circ} \text { de comuni- } \\
\text { caciones [3] }\end{array}$ & $\begin{array}{c}\text { Ratio } \\
{[\mathbf{3} \mathbf{2}]}\end{array}$ \\
\hline Universidad Autónoma de Barcelona & 7 & 3 & 0,42 \\
\hline Universidad de Santiago de Compostela & 7 & 5 & 0,71 \\
\hline Universidad de Barcelona & 6 & 5 & 0,83 \\
\hline Universidad de Valencia & 3 & 3 & 1,0 \\
\hline Universidad Autónoma de Madrid & 3 & 2 & 0,67 \\
\hline Universidad Complutense de Madrid & 3 & 2 & 0,67 \\
\hline Universidad de Sevilla & 3 & 2 & 0,67 \\
\hline Universidad de Islas Baleares & 2 & 2 & 1,0 \\
\hline Universidad de Las Palmas de Gran Canarias & 2 & 1 & 0,50 \\
\hline Universidad de Girona & 2 & 1 & 0,50 \\
\hline Universidad de Castilla-La Mancha & 2 & 1 & 0,50 \\
\hline Universidad de Granada & 2 & 2 & 1,0 \\
\hline Universidad Politécnica de Madrid & 1 & 1 & 1,0 \\
\hline Total & $\mathbf{4 3}$ & $\mathbf{3 0}$ & $\mathbf{0 , 6 9}$ \\
\hline
\end{tabular}

Fuente: Censo de visitantes al stand del Comité Español de la UGI (Elaboración propia).

6 No disponemos de datos sobre la participación de colegas españoles en las reuniones de trabajo programadas por muchas comisiones en la semana previa a la celebración de la sesión principal del Congreso. A partir de noticias fragmentarias deducimos que ésta ha sido también relevante, pero supondría un esfuerzo de búsqueda informativa inasumible para redactar esta nota el rastreo de datos más precisos. Ya ha sido laborioso acumular la información necesaria para describir detalladamente en este apartado de la reseña la aportación de los geógrafos españoles pues los organizadores alemanes sólo han proporcionado datos sistematizados a través del programa sobre el calendario de las sesiones y la relación de los intervinientes. Ha sido preciso, por ello, hacer un paciente acopio, utilizando esta fuente, de los nombres, universidades de procedencia y título de la aportación con el que poder confeccionar un inventario exhaustivo de los geógrafos españoles que de forma individual o colectiva han firmado una comunicación al congreso. Mucho menos detallado ha podido ser el conocimiento de los inscritos, dato que no tiene por qué coincidir con la información anterior debido a los artículos de firma colectiva o a las ausencias imprevistas de los inscritos. 
de todas las sesiones del congreso. El saldo resultante da un total de 63 autores (58 procedentes de universidades y cinco de otras instituciones investigadoras), que en conjunto han presentado 37 comunicaciones, lo que da una media de dos autores por comunicación. Estos datos multiplican por tres el número de comunicaciones y de autores presentes en el anterior congreso de Túnez.

Es muy expresivo del interés despertado por esa edición de los congresos internacionales de Geografía que miembros de 18 universidades hayan participado en las comunicaciones presentadas, aunque la distribución no se precisamente homogénea, como se desprende del Cuadro 3. Así, cuatro universidades acaparan casi la mitad de los participantes (Barcelona y Autónoma de Barcelona, Santiago y Valencia), exactamente un 44,8\%; las dos mayores universidades de Madrid con 12 de los autores sólo alcanzan un modesto 13,8 \%. A partir de aquí el reparto se atomiza sin base para demasiadas matizaciones.

Además, fueron presentadas siete comunicaciones firmadas por 22 autores procedentes de distintas universidades y centros de investigación según la combinación desarrollada en el cuadro 4)

Cuadro 4

COMUNICACIONES PRESENTADAS AL CONGRESO DE COLONIA REALIZADAS EN COLABORACIÓN ENTRE UNIVERSIDADES Y CENTROS DE INVESTIGACIÓN

\begin{tabular}{|c|c|c|c|}
\hline Universidades e instituciones [1] & \begin{tabular}{|c|}
$N^{\circ}$ de autores \\
{$[2]$}
\end{tabular} & $\begin{array}{c}\mathbf{N}^{\circ} \text { de comu- } \\
\text { nicaciones [3] }\end{array}$ & $\begin{array}{l}\text { Ratio } \\
{[3 / 2]}\end{array}$ \\
\hline Universidad Complutense de Madrid & 1 & \multirow{2}{*}{2} & \multirow{2}{*}{1,0} \\
\hline Universidad de León & 1 & & \\
\hline Universidad San Jorge & 1 & \multirow{2}{*}{1} & \multirow{2}{*}{0,50} \\
\hline Centro de Desarrollo de Energías Renovables & 1 & & \\
\hline Universidad de Las Palmas de Gran Canarias & 1 & \multirow{2}{*}{1} & \multirow{2}{*}{0,50} \\
\hline Asociación de Geógrafos Españoles & 1 & & \\
\hline Universidad de Islas Baleares & 2 & \multirow{3}{*}{1} & \multirow{3}{*}{0,17} \\
\hline Universidad de Valencia & 3 & & \\
\hline $\begin{array}{l}\text { SOCIB, Balearic Islands Coastal Observing and } \\
\text { Forecasting System }\end{array}$ & 1 & & \\
\hline Universidad San Jorge & 1 & \multirow{4}{*}{1} & \multirow{4}{*}{0,2} \\
\hline Universidad de Salamanca & 1 & & \\
\hline Universidad de Zaragoza & 2 & & \\
\hline Universidad Politécnica de Madrid & 1 & & \\
\hline CCHS-CSIC Madrid & 2 & \multirow{4}{*}{1} & \multirow{4}{*}{0,2} \\
\hline Universidad de Zaragoza & 1 & & \\
\hline Universidad Autónoma de Madrid & 1 & & \\
\hline Universidad de Málaga & 1 & & \\
\hline Total & 22 & 7 & $\mathbf{0 , 3 1}$ \\
\hline
\end{tabular}

Fuente: Censo de visitantes al stand del Comité Español de la UGI (Elaboración propia). 
En cuanto a las temáticas, también destaca la enorme dispersión de las aportaciones presentadas, no pudiendo destacarse ninguna en particular de manera clara, ya que tan sólo Urbanización y Cambio Demográfico supera el umbral de cinco contribuciones, lo cual indica la diversificación de las preocupaciones científicas de los geógrafos españoles, pero también es un fiel reflejo de la propia dispersión temática de nuestra disciplina.

Cuadro 5

TEMÁTICAS EN QUE SE INCARDINAN LAS APORTACIONES DE LOS GEÓGRAFOS ESPAÑOLES AL CONGRESO DE COLONIA

\begin{tabular}{|l|c|c|}
\hline Tema & Contribución & Porcentaje \\
\hline Climatología & 1 & 2,7 \\
\hline Sistemas costeros & 2 & 5,4 \\
\hline Medio ambiente de las regiones polares & 1 & 2,7 \\
\hline Toponimia & 2 & 5,4 \\
\hline Geografía Cultural & 1 & 2,7 \\
\hline Transformaciones urbanas emergentes & 1 & 2,7 \\
\hline Género y geografía & 1 & 2,7 \\
\hline Geografía de la gobernanza & 1 & 2,7 \\
\hline Geografía del turismo, ocio y cambio global & 3 & 8,1 \\
\hline Cambio global y globalización & 2 & 5,4 \\
\hline Cambio global y movilidad humana & 2 & 5,4 \\
\hline Historia de la geografía & 2 & 5,4 \\
\hline Cambios en el uso y ocupación del suelo & 1 & 2,7 \\
\hline Desarrollo local & 1 & 2,7 \\
\hline Programa Renacimiento del Mediterráneo & 1 & 2,7 \\
\hline Geografía de la Población & 2 & 5,4 \\
\hline Sociedad y medio ambiente & 3 & 8,1 \\
\hline Sostenibilidad de los sistemas rurales & 2 & 5,4 \\
\hline Transporte y geografía & 3 & 8,1 \\
\hline Urbanización y cambio demográfico & 5 & 13,5 \\
\hline Total & $\mathbf{3 7}$ & $\mathbf{1 0 0 , 0}$ \\
\hline
\end{tabular}

Fuente. Programa del Congreso de Colonia 2012 (Búsqueda y sistematización propia).

Nota: Los nombres de las temáticas se corresponden básicamente con los de las comisiones de la UGI.

\section{REFLEXIONES FINALES}

Resulta alentador comprobar que, a pesar de la considerable reducción de medios disponibles en un contexto de crisis como en el que nos hallamos, con su negativo efecto tanto sobre los individuos como sobre las instituciones, la presencia española en el Congreso de Colonia se ha reforzado en cantidad de participantes y de contribuciones, muchas de ellas presentadas por varios autores; esta observación refleja la práctica creciente del trabajo en equipo, que va adquiriendo carta de naturaleza en nuestros centros de investigación. Igual- 
mente es alentadora la excelente acogida dispensada a la aportación corporativa del Comité Español en formato $\mathrm{CD}$, que ha ganado en manejo y en atractivo por la calidad de su diseño y por la incorporación del color, así como en accesibilidad, al haber sido colgado en las webs institucionales y de equipos de investigación. La parte negativa de la situación se la lleva la reducción de los medios materiales y humanos puestos a disposición del stand, lo que ha limitado las posibilidades de exhibición de bibliografía, documentación y cartografía de congresos anteriores, con la consiguiente pérdida de atractivo y de imagen frente a otros comités e instituciones presentes en Colonia. Bien es verdad que se ha intentado compensar con el entusiasmo y dedicación de los miembros del comité presentes en Colonia y algunos/ as generosos colegas que han colaborado en las tareas de presencia en el stand y de difusión del CD con la aportación española a Colonia.

Con la vista puesta en la década 2012-2022, en que los geógrafos de todo el mundo estamos convocados a ocho eventos geográficos internacionales entre congresos y conferencias regionales, sería muy conveniente realizar una seria reflexión sobre la presencia de la geografía española en el seno de la Unión Geográfica Internacional como colectivo de geógrafos y como miembros de una comunidad cultural y lingüística de proyección mundial, justamente ahora que a nivel institucional (Comité Ejecutivo de la UGI) ésta ha dejado prácticamente de existir. Quizá esta reflexión nos anime a articular formas de participación más activa y organizada en este mundo globalizado en todas las facetas de la vida, la economía y, por supuesto, de la ciencia y que de tales presupuestos también salga reforzada la calidad de nuestro mensaje y de nuestras aportaciones a la ciencia geográfica mundial.

Agradecimientos: El autor de esta reseña desde agradecer a Marisela Pilquimán, doctoranda en Geografía Humana, por su eficaz ayuda en la explotación de la información contenida en el Censo de Visitantes y en el Programa del Congreso, y a Nicolás Delmonte, colaborador de proyecto de investigación, por su apoyo informático y cartográfico.

Madrid, noviembre de 2012 